\title{
Calidad fisiológica de semillas y variabilidad entre plantas en una población de arroz
}

\section{Seeds physiological quality and variability among plants in populations of rice}

\author{
Luis Osmar Braga Schuch ${ }^{1}$, Igor Kohls ${ }^{2}$, Andreia da Silva Almeida ${ }^{1}$, Cristiane Deuner ${ }^{*}$, Jerffeson Araujo Cavalcante $^{l}$
}

Resumen: La calidad fisiológica de la semilla podría afectar el rendimiento de granos de arroz. Las diferencias en la calidad fisiológica de las semillas pueden causar la reducción y desuniformidad de la emergencia en el campo, acarreando una diferencia en el crecimiento inicial de las plantas, y consecuentemente afectando el cultivo y su rendimiento. Se realizo un experimento con el objetivo de evaluar la distribución espacial y la variabilidad en la población entre las plantas de arroz, en función de la calidad fisiológica de las semillas. Fueron utilizados nueve lotes de semillas de la cultivar BR-IRGA 410, siendo tres de alta, tres de media y tres de baja calidad fisiológica. Cada bloque consistió de nueve líneas de 5,5 metros de longitud, separadas por 0,12 metros. Se evaluó el número de fallas consecutivas y la variabilidad entre las plantas a través del coeficiente de variación de la altura de plantas y de la materia seca. El uso de las semillas con baja calidad fisiológica proporcionó mayor desuniformidad en la distribución espacial de la planta en el campo, y una mayor variabilidad entre las plantas que componen la población, lo que puede proporcionar un aumento en la competencia intra-especifica.

Palabras clave: Oryza sativa L., fallas, desuniformidad, vigor

\begin{abstract}
Several authors try to relate the physiological quality of seeds with grain yield of rice. Differences in the physiological quality of seeds can cause reduction and uniformity of emergence in the field, leading to differential on early seedling growth, and consequently affecting the culture and your yield. An experiment was conducted to evaluate the spatial distribution and variability in populations of rice plants, depending on the physiological quality of seeds. Were used nine seed lots of cultivar BR-IRGA 410, three lots of high, three lots of medium and three lots of low physiological quality. Each block was composed of nine lines of 5.5 meters in length, spaced 0.12 meters. We evaluated the number of consecutive failures in emergence, and plant variability through the coefficient of variation of plant height and dry matter. The use of seed with low physiological quality provides increased uniformity in the spatial distribution of plants in the field, and greater variability from plants comprising the population, which can provide an increase in intra-specific competition.
\end{abstract}

Key words: Oryza sativa L., faluires, non uniformity, vigor

\footnotetext{
*Autor para correspondência

Recebido para publicação em 12/05/2016; aprovado em 28/06/2016

${ }^{1}$ Departamento de Fitotecnia, UFPel/FAEM, Caixa Postal 354,96001-970. Capão do Leão-RS, Brasil. E-mail: cdeuner@ yahoo.com.br

${ }^{2}$ Instituto Rio Grandense do Arroz, Porto Alegre-RS, Brasil.
} 


\section{INTRODUCCIÓN}

En Brasil fueron producidas 12,435 millones de toneladas de arroz en la zafra 2014/15, en una área de 2,29 millones de hectáreas. Solamente en Rio Grande do Sul fueron producidos 8,624 millones de toneladas en una área de 1,12 millones de hectáreas (CONAB, 2015). Para obtener buenos rendimientos de los cultivos, es necesario que las semillas presenten una buena calidad, tanto genética, como fisiológica y especialmente sanitaria, porque son estas las características que determinan la capacidad de una semilla originar una planta productiva (TELÓ et al., 2012). El vigor de las semillas tiene efecto en las primeras etapas de crecimiento de las plantas, definiendo el mejor uso de los recursos como agua, luz y nutrientes y la competencia con malezas.

Las semillas que presentan mayor calidad fisiológica permiten un rápido establecimiento del cultivo, debido a la mayor velocidad en los procesos metabólicos que resulta en mayores tasas de crecimiento de plantas con mayor tamaño inicial (SCHUCH et al., 1999), reduciendo posibles riesgos de perdida en la producción por competencia. Melo et al. (2006), en arroz de riego, encontraron que las comunidades de plantas procedentes de semillas de alto vigor presentaron rendimiento superior al $20 \%$ en comparación con las comunidades de plantas instaladas a partir de semillas de bajo vigor.

Mucho ha sido discutido sobre las causas de la reducción de la productividad de los cultivos por el uso de semillas de baja calidad fisiológica. Varios autores asocian el bajo vigor de las semillas con reducción en la velocidad y en la emergencia total, desuniformidad de germinación, reducción del tamaño inicial de las plantas, de la producción de materia seca, del área foliar y de las tasas de crecimiento del cultivo (SCHUCH et al., 2000; HÖFS et al., 2004; LUDWIG et al., 2009). Una causa probable de la reducción del rendimiento de granos cuando se usan lotes de semillas con menor calidad fisiológica, es por la posible desuniformidad de la distribución espacial de las plantas dentro del cultivo, ocasionada por la variabilidad del propio lote de semillas, provocando competencia intraespecífica, lo que resulta en un lento crecimiento inicial que pude persistir hasta la cosecha. De acuerdo con Cantarelli et al. (2015), semillas de baja calidad fisiológica causan una disminución en la supervivencia de las plantas en el campo y el aumento de la variabilidad entre las plantas en la comunidad vegetal.

De esta forma, el presente trabajo tuvo como objetivo evaluar la distribución espacial y la variabilidad entre plantas de arroz a lo largo de la línea de siembra, en función de la calidad fisiológica de las semillas.

\section{MATERIALES Y MÉTODOS}

El experimento fue realizado en el Área Experimental y Didáctica del Departamento de Fitotecnia de la Facultad de Agronomía "Eliseu Maciel" de la Universidad Federal de Pelotas (FAEM/UFPel), localizada en el municipio de Capão do Leão -RS. Fueron utilizados nueve lotes de semillas del cultivar BR-IRGA 410 en tres niveles diferentes de calidad fisiológica, que son: alta, media y baja calidad fisiológica. Los lotes de alta calidad fisiológica fueron obtenidos de productores de semillas de arroz, mientras que los lotes de media y baja calidad fisiológica fueron envejecidos artificialmente por el método de envejecimiento acelerado modificado, a partir de los lotes de alta calidad, siendo utilizados periodos de tiempo y temperaturas determinadas preliminarmente para conseguir los niveles deseados de calidad fisiológica. La calidad fisiológica fue reducida en torno de $10 \%$ y $20 \%$ de la germinación para los lotes de media y baja calidad, en relación a lotes de alta calidad fisiológica, utilizando las combinaciones de $45^{\circ} \mathrm{C} / 136 \mathrm{~h}$ e 45 ${ }^{\circ} \mathrm{C} / 168 \mathrm{~h}$, respectivamente. Los tratamientos incluyen los niveles de calidad fisiológica descritos anteriormente, cada uno compuesto por la media de tres lotes. Después de la diferenciación de los lotes, las semillas fueron sometidas a los análisis de germinación y primer conteo de germinación (BRASIL, 2009). También fueron realizados ensayos de frio y envejecimiento acelerado.

Para la evaluación de la distribución espacial de las plantas en el campo, cada lote de semillas fue sembrado de forma equidistante a lo largo de una línea de 5,5 metros de longitud con ayuda de regla graduada, con espaciamiento entre líneas de 0,12 metros. La densidad de siembra fue corregida por el potencial germinativo de cada lote, con el fin de obtener una población de 300 semillas viables por $\mathrm{m}^{-2}$, de modo que los espaciamientos entre los puntos de siembra fueron de 2,6 cm; 2,3 cm y 2,0 cm para los lotes de alta calidad, media y baja calidad fisiológica, respectivamente. Se entiende por punto de siembra al local ocupado por una semilla en la línea de siembra. El número de semillas no germinadas forma consecuentemente una falla en la línea de siembra, siendo esta utilizada para evaluar la distribución espacial a los 21 días después de la emergencia (DDE), en una, dos, tres, cuatro, cinco y seis fallas consecutivas. Para la evaluación de la variabilidad entre plantas fueron realizados análisis de emergencia en campo e índice de velocidad de emergencia para los diferentes lotes, utilizando metodologías propuestas por Nakagawa (1994). Para esto, fueron marcadas dos repeticiones de 100 semillas en todas las líneas de siembra. También a los $21 \mathrm{DDE}$, se evaluó la longitud de la parte aérea de 40 plantas de cada parcela. A los 41 DDE fueron colectadas 40 plantas de cada parcela y evaluadas la longitud de la parte aérea y la masa de la materia seca (MMS). Con los valores obtenidos individualmente, fue determinado el coeficiente de variación (CV) y estimada la variabilidad entre las plantas, en función del nivel de calidad fisiológica de las semillas, y adicionalmente se analizaron las medias determinadas para cada variable.

El diseño experimental fue de bloques completos al azar, con cinco repeticiones. Los datos experimentales fueron sometidos a análisis de variancia y los efectos de tratamiento evaluados por test de Fisher. Para las comparaciones de las medias dentro de los tratamientos, fue utilizado el test de Duncan al $5 \%$ de probabilidad. Para esto fue utilizado el programa de estadística SANEST (ZONTA; MACHADO, 1986).

\section{RESULTADOS Y DISCUSIÓN}

En la Tabla 1 son presentados los resultados del análisis de germinación, primer conteo de germinación, ensayo de frio, envejecimiento acelerado, índice de velocidad de emergencia y emergencia en campo, donde se pueden observar diferencias entre los niveles de calidad fisiológica de las semillas. Resultados semejantes también fueron constatados por Albuquerque y Carvalho (2003), Höfs et al. (2004) y Ludwig et al. (2008). Según Schuch et al. (1999), la 
reducción en el nivel de vigor de las semillas aumentó el tiempo necesario para la emergencia de las raíces primarias alrededor de 1 a 2 días en semillas de avena negra, probablemente ocasionado por la reducción en la degradación de las sustancias de reserva, trasporte y síntesis de nuevos compuestos en el eje embrionario cuando utilizadas semillas de bajo vigor. Autores relatan reducción en la germinación en campo, índice de velocidad de germinación y velocidad de germinación, con la reducción del vigor de las semillas, además de mayor desuniformidad de emergencia ( $\mathrm{SCHUCH}$ et al., 2000; HÖFS et al., 2004).

Tabla 1 - Germinación (G), primer conteo de germinación (PCG), ensayo de frio (TF), envejecimiento acelerado (EA), índice de velocidad de germinación (IVE), velocidad de germinación (VE) y emergencia en campo (E), en tres niveles de calidad fisiológica de semillas de arroz del cultivar -IRGA 410 .

\begin{tabular}{lccccccc}
\hline \multirow{2}{*}{ Nivel } & $\begin{array}{c}\mathrm{G} \\
(\%)\end{array}$ & $\begin{array}{c}\text { PCG } \\
(\%)\end{array}$ & $\begin{array}{c}\text { TF } \\
(\%)\end{array}$ & $\begin{array}{c}\text { EA } \\
(\%)\end{array}$ & $\begin{array}{c}\text { IVE } \\
\left(\text { Pl.día }^{-1}\right)\end{array}$ & $\begin{array}{c}\text { VE } \\
(\text { día })\end{array}$ & $\begin{array}{c}\text { E } \\
(\%)\end{array}$ \\
\hline Alta & $96 \mathrm{a}$ & $92 \mathrm{a}$ & $87 \mathrm{a}$ & $88 \mathrm{a}$ & $15,16 \mathrm{a}$ & $6,0 \mathrm{~b}$ & $89 \mathrm{a}$ \\
Media & $85 \mathrm{~b}$ & $81 \mathrm{~b}$ & $79 \mathrm{~b}$ & $80 \mathrm{~b}$ & $12,39 \mathrm{~b}$ & $6,5 \mathrm{a}$ & $78 \mathrm{~b}$ \\
Baja & $76 \mathrm{c}$ & $72 \mathrm{c}$ & $71 \mathrm{c}$ & $71 \mathrm{c}$ & $11,19 \mathrm{c}$ & $6,5 \mathrm{a}$ & $72 \mathrm{c}$ \\
\hline Media & 86 & 82 & 79 & 80 & 12,91 & 6,3 & 79,99 \\
\hline $\mathrm{CV}(\%)$ & 3,29 & 2,47 & 3,25 & 2,92 & 10,22 & 7,84 & 4,54 \\
\hline
\end{tabular}

Medias seguidas por la misma letra no difieren entre si por el test de Duncan al $5 \%$ de probabilidad.

Plantas provenientes de semillas de alta calidad fisiológica presentaron mayor tamaño a los 21 DDE comparándolas con los otros niveles. De la misma forma, a los 40 DDE, plántulas provenientes de semillas de alta calidad presentaron mayor desarrollo, sin embargo, no fueron estadísticamente diferentes de las semillas de media calidad (Tabla 2). Plantas de mayor altura relacionadas con semillas de mayor calidad también fueron observadas en soja (VANZOLINI; CARVALHO, 2002; SCHUCH et al., 2009). Por su parte, Höfs et al. (2004) no observaron diferencias entre los niveles de calidad fisiológica de las semillas de arroz en relación a la altura de las plantas.

Tabla 2 - Longitud de la parte aérea (LLPA) a los 21 y 40 días después de la emergencia (DDE), masa de la materia seca a los 40 días después de la germinación y variabilidad entre plantas evaluada por el coeficiente de variación (CV), en tres niveles de calidad fisiológica de semillas de arroz de la cultivar IRGA 410.

\begin{tabular}{|c|c|c|c|c|c|c|}
\hline \multirow{3}{*}{ Nivel } & \multicolumn{4}{|c|}{ LLPA } & \multirow{2}{*}{\multicolumn{2}{|c|}{$\frac{\text { MMS }}{40 \mathrm{DDE}}$}} \\
\hline & \multicolumn{2}{|c|}{$21 \mathrm{DDE}$} & \multicolumn{2}{|c|}{$40 \mathrm{DDE}$} & & \\
\hline & $(\mathrm{cm})$ & $\mathrm{CV}$ & $(\mathrm{cm})$ & $\mathrm{CV}$ & (g) & $\mathrm{CV}$ \\
\hline Alto & $15,9 \mathrm{a}$ & $14,0 \mathrm{~b}$ & $32,4 \mathrm{a}$ & $10,7 \mathrm{~b}$ & $0,85 \mathrm{a}$ & $34,1 \mathrm{~b}$ \\
\hline Medio & $14,9 \mathrm{~b}$ & $15,1 \mathrm{ab}$ & $31,7 \mathrm{a}$ & $11,5 \mathrm{ab}$ & $0,77 \mathrm{~b}$ & $36,3 \mathrm{~b}$ \\
\hline Bajo & $14,5 \mathrm{~b}$ & $16,0 \mathrm{a}$ & $30,3 \mathrm{~b}$ & $12,5 \mathrm{a}$ & $0,68 \mathrm{c}$ & $41,2 \mathrm{a}$ \\
\hline Media & 15,17 & 15,01 & 31,49 & 11,57 & 0,77 & 37,23 \\
\hline $\mathrm{CV}(\%)$ & 6,98 & 13,41 & 5,1 & 16,98 & 10,78 & 17,27 \\
\hline
\end{tabular}

Medias seguidas por la misma letra no difieren entre si por el test de Duncan al $5 \%$ de probabilidad.

Semillas de alta calidad fisiológica también ocasionaron una mayor cantidad de MMS a los 40 DDE, diferenciándose de los niveles de media y baja calidad fisiológica, superior a 9 y $20 \%$, respectivamente (Tabla 2). Resultados semejantes fueron encontrados por Schuch et al. (2000), Kolchinski et al. (2006), Melo et al. (2006), Ludwig et al. (2008) y Mielezrski et al. (2008b). De acuerdo con Schuch et al. (2000), el uso de semillas de alto vigor resulta en plantas con un tamaño inicial superior y con altas tasas de crecimiento a lo largo del desarrollo del cultivo, aumentando así el acúmulo de MMS.

En la tabla 2 son presentadas las medias de los CV para longitud de la parte aérea a los 21 y 40 DDE y MMS a los 41 DDE. En todas las variables, las semillas de alta calidad fisiológica presentaron plantas con mayor uniformidad dentro de las poblaciones, comparándolas con las plantas de baja calidad, sin embargo, semillas de alta calidad no se diferenciaron de las de media calidad fisiológica. Este resultado fue similar al encontrado por Kolchinski et al. (2005) al estudiar diferencias de vigor entre semillas dentro de un mismo lote de semillas. Estos autores constataron que esas diferencias fueron más acentuadas en los lotes de baja calidad fisiológica.

Lotes de baja calidad fisiológica presentan una mayor variabilidad de calidad entre las semillas que componen el lote, provocando así mayor desuniformidad en la germinación en campo y originando plantas de menor tamaño inicial, lo cual proporciona menores tasas de crecimiento del cultivo (HÖFS et al., 2004; KOLCHINSKI et al., 2005; LUDWIG et al., 2009). Estas diferencias ocasionan una alta variabilidad entre plantas, donde las plantas que germinaron primero dominan las plantas con emergencia tardía, derivando en mayor competencia por factores ambientales. De otro modo, poblaciones más uniformes proporcionan mayores rendimientos de granos por reducir el efecto de sombreamiento de las plantas emergidas tardíamente.

La mayor uniformidad en la emergencia que ocurre en lotes de mayor calidad fisiológica, contribuye a que las plantas emergidas posteriormente no consigan acompañar el desarrollo de las que germinaron primero, por lo que las plantas que se originan de semillas de baja calidad son menos competitivas por espacio, luz, agua y nutrientes (SCHUCH et al., 2000; LUDWIG et al., 2008; BRAZ; ROSSETO, 2009; LUDWIG et al., 2009). Esto posibilita la intensificación de la competencia intraespecífica dentro de las poblaciones de plantas originadas de semillas de calidad fisiológica más baja, proporcionado efectos de dominancia entre las plantas originadas de diferentes niveles de calidad de semillas dentro de poblaciones, con probables efectos sobre el rendimiento del grano.

Por los resultados presentados en la Figura 1, se observa que el número de fallas de un único punto de siembra fue relativamente alto en semillas de baja calidad, mientras que semillas con alta calidad fisiológica presentan una menor cantidad de eventos (fallas), y las de media calidad una posición intermediaria. Las semillas de alta calidad fisiológica tuvieron en torno de 10,5 eventos, en cuanto que las semillas de media y baja calidad fisiológica presentan en torno de $15 \mathrm{y}$ 18 eventos, un aumento en torno de $30 \%$ y $42 \%$, respectivamente, a lo largo de la línea de 5,5 metros. De manera similar, cuando hubo dos fallas consecutivas, los niveles presentaban diferencias entre sí, pero con una reducción significativa del número de eventos, en comparación con la anterior. Con el aumento del número de fallas consecutivas, permaneció la tendencia decreciente de la ocurrencia de los eventos, sin embargo, los niveles de media a 
baja calidad fisiológica no difirieron entre sí, y difirieron de las semillas de alta calidad con valores superiores.

Figura 1 - Distribución espacial de las plantas de arroz del cultivar BR_IRGA 410 a lo largo de 5,5 metros de la línea de siembra, de lotes de semillas de alta, media y baja calidad fisiológica.

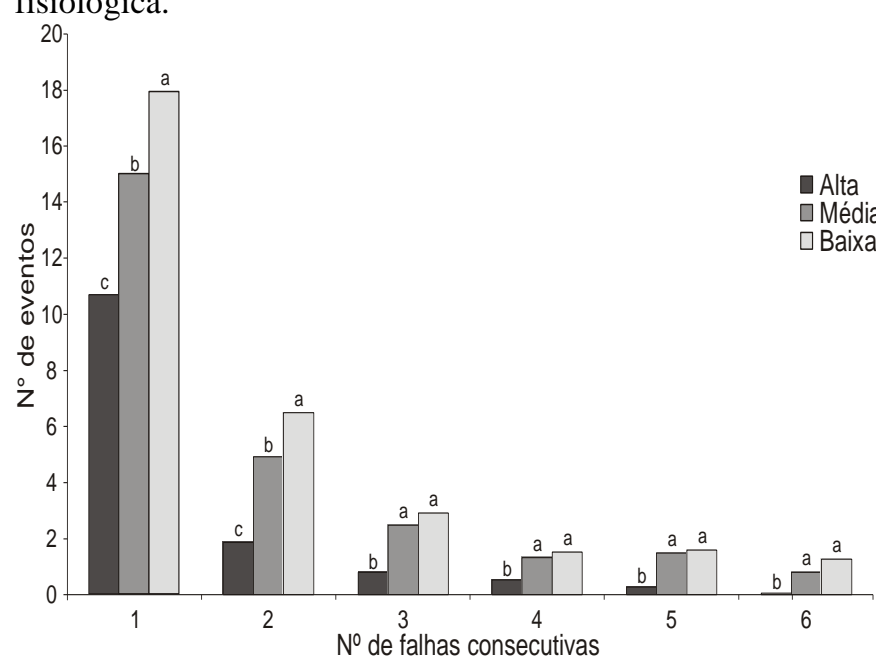

El deterioro de las semillas puede ser considerado como una de las causas de las fallas en la germinación o mismo en la reducción de la velocidad de germinación, además de disminuir la resistencia a estreses ambientales durante la germinación en las primeras etapas de desarrollo, lo que provoca fallas en la población de plantas en el campo. Por ese motivo es importante el uso de semillas de alto vigor para promover el rápido y uniforme establecimiento de la población de plantas a campo.

También se observó que para las fallas de 2, 3 y 4 puntos de siembra consecutivos, se encontraron en torno de 2; 0,8 y 0,5 eventos en el nivel de alta calidad fisiológica, mientras que el nivel de baja calidad fisiológica se observaron en torno de 6,3; 2,8 y 1,5 eventos, respectivamente. El nivel de alta calidad fisiológica prácticamente no presentó fallas de 5 y 6 puntos consecutivos de siembra, en tanto que el de baja calidad fisiológica presentó en torno de 1,6 y 1,3 eventos, respectivamente (Figura 1).

En los 5,5 metros lineares de siembra, fueron sembrados 214; 242 y 270 semillas y ocurrieron 14; 26 y 32 fallas respectivamente para los niveles de alta, media y baja calidad fisiológica. De esta forma se constató que la calidad del lote de semillas afecto la distribución espacial de las plantas dentro de la comunidad de arroz, evidenciando que la práctica de corregir la densidad de siembra utilizando los valores de la germinación y/o del vigor de los lotes de semillas pueden ser útil en establecer una población de plantas adecuada o más próxima de lo deseado. En tanto, la distribución espacial de las plantas en la comunidad queda comprometida, debido a que poblaciones provenientes de semillas de bajo vigor presentan mayor número de fallas, fallas grandes y una mayor densidad de plantas en los locales donde no ocurren fallas, durante la corrección de germinación. Luego, la utilización de las semillas de alta calidad fisiológica, proporciona una distribución uniforme de las plantas en las líneas de siembra y consecuentemente un aumento en el rendimiento de los granos, como fue observado por diversos investigadores (MIELEZRSKI et al., 2008a; MIELEZRSKI et al., 2008b; SCHUCH et al., 2009).

\section{CONCLUSIONES}

La utilización de semillas de menor calidad fisiológica causa disminución, atraso y menor uniformidad de germinación en campo, además de reducir la estatura y la masa de la materia seca de las plantas de arroz.

La utilización de semillas de baja calidad fisiológica proporciona mayor desuniformidad en la distribución espacial de plantas en el campo y alta variabilidad entre las plantas que componen la población.

\section{REFERENCIAS BIBLIOGRÁFICAS}

ALBUQUERQUE, M. C. F.; CARVALHO, N. M. Effects ofthe environmental stress on the emergence of sunflower(Helianthus annuus L.), soybean (Glycine max L. Merril)and maize (Zea mays L.) seeds with different levels of vigor.Seed Science and Technology, v.31, p.465-479,2003.

BRASIL. Ministério da Agricultura, Pecuária e Abastecimento. Regras para Análise de Sementes. Ministério da Agricultura, Pecuária e Abastecimento - Secretaria de Defesa Agropecuária. Brasília: Mapa/ACS, 2009. 399p.

BRAZ, M. R. S.; ROSSETO, C. A. V. Estabelecimento de plântulas e desempenho de plantas em resposta ao vigor dos aquênio de girassol. Ciência Rural, v.39, n.7, p.1997-2003, 2009.

CONAB. Ministério da Agricultura Pecuária e Abastecimento. Safra 2011/2012: Acompanhamento da safra brasileira - quarto levantamento. Brasília: CONAB, 2012. Acessado em 01mar. 2012. Disponível em: <http://www.conab.gov.br>.

HÖFS, A. SCHUCH, L. O. B.; PESKE, S. T.; BARROS, A. C. S. A. Emergência e crescimento de plântulas de arroz em resposta à qualidade fisiológica de sementes. Revista Brasileira de Sementes, v.26, n.1, p.92-97, 2004.

KOLCHINSKI, E. M.; SCHUCH, L. O. B.; PESKE, S. T. Vigor de sementes e competição intra-específica em soja. Ciência Rural, v.35, n.6, p.1248-1256, 2005.

KOLCHINSKI, E. M.; SCHUCH, L. O. B.; PESKE, S. T. Crescimento inicial de soja em função do vigor das sementes. Revista Brasileira de Agrociência, v.12, n.2, p.163-166, 2006.

LUDWIG, M. P. SCHUCH, L. O. B.; LUCCA FILHO, O. A.; AVELAR, S. A. G.; MIELEZRSKI, F.; PANOZZO, L. E.; OLIVO, M.; SEUS, R. Desempenho de plantas de feijão originadas de lotes de sementes com diferentes níveis de qualidade fisiológica. Revista da Faculdade de Zootecnia, Veterinária e Agronomia, v.15, n.2, p.44-52, 2008.

LUDWIG, M. P.; SCHUCH, L. O. B.; LUCCA FILHO, O. A.; AVELAR, S. A. G.; MIELEZRSKI, F.; OLIVEIRA, S.; CRIZEL, R. L. Desempenho de sementes e plantas de milho híbrido de lotes de sementes com alta e baixa qualidade fisiológica. Revista Brasileira de Milho e Sorgo, v.8, n.1, p.83-92, 2009. 
MELO, P. T. B. S.; SCHUCH, L. O. B.; ASSIS, F. N.; CONCENÇO, G. Comportamento individual de plantas originadas de sementes com diferentes níveis de qualidade fisiológica em populações de arroz irrigado. Revista Brasileira de Sementes, v.28, p.84-94, 2006.

MIELEZRSKI, F.; SCHUCH, L. O. B.; PESKE, S. T.; PANOZZO, L. E.; PESKE, F. B.; CARVALHO, R.R. Desempenho individual e de populações de plantas de arroz híbrido em função da qualidade fisiológica das sementes. Revista Brasileira de Sementes, v.30, n.3, p.86-94, 2008a.

MIELEZRSKI, F.; SCHUCH, L. O. B.; PESKE, S. T.; PANOZZO, L. E.; CARVALHO, R. R.; ZUCHI, J. Desempenho em campo de plantas isoladas de arroz híbrido em função da qualidade fisiológica das sementes. Revista Brasileira de Sementes, v.30, n.3, p.139-144, 2008b.

NAKAGAWA, J. Testes de vigor baseados na avaliação das plântulas. In: VIEIRA, R.D.; CARVALHO, N.M. (Ed.). Testes de vigor em sementes. Jaboticabal: FUNEP, 1994. p.49-86.

SCHUCH, L.O.B.; NEDEL, J.L.; ASSIS, F.N.; MAIA, M.S. Crescimento em laboratório de plântulas de aveia-preta (Avena strigosaSchreb.) em função do vigor das sementes. Revista Brasileira de Sementes, v.21, n.1, p.229-234, 1999.

SCHUCH, L. O. B.; NEDEL, J. L.; ASSIS, F. N.; MAIA, M S. Vigor de sementes e análise de crescimento de aveia preta. Scientia Agrícola, v.57, n.2, p.305-312, 2000.

SCHUCH, L. O. B.; KOLCHINSKI, E. M.; FINATTO, J. A Qualidade fisiológica da semente e desempenho de plantas isoladas em soja. Revista Brasileira de Sementes, v.31, n.1, p.144-149, 2009.

VANZOLINI, S.; CARVALHO, N. M. Efeito do vigor de sementes de soja sobre o seu desempenho em campo. Revista Brasileira de Sementes, v.24, n.1, p.33-41, 2002.

ZONTA, E. P.; MACHADO, A. A. Sistema de análise estatística para microcomputadores - SANEST. Pelotas: UFPel, Instituto de Física e Matemática, Departamento de Estatística, 1986, 150p. 